\title{
Shape Memory and Superelasticity Best Paper Award
}

\section{(C) ASM International 2016}

To recognize exceptional contributions to Shape Memory and Superelasticity we are introducing the Shape Memory and Superelasticity Best Paper Award.

The award, in addition to the recognition, includes a plaque and $\$ 1000$ worth of ASM International products and services.

Selection of the best paper will be conducted annually by the Editor and Associate Editors of Shape Memory and
Superelasticity. The winning paper will be chosen through a ranking process that includes all of the papers published in the volume of the previous year.

The winning papers will be recognized at the next Shape Memory and Superelastic Technologies Conference. 